\title{
Application Ultrasound Ablation (HIFU) in the Treatment of Benign Tumors of the Mammary Glands
}

\author{
S. Imankulov, K. Rustemova ${ }^{*}$ Z. Seidagaliyeva, N. Aykumbekov \\ National Scientific Medical Centre, Department of Surgical Diseases, Astana Medical University, Kazakhstan
}

Copyright (C) 2015 by authors, all rights reserved. Authors agree that this article remains permanently open access under the terms of the Creative Commons Attribution License 4.0 International License

\begin{abstract}
The main method of treatment of breast fibroadenomas, until recently, was considered a surgical. However, there is research the authors of a new non-invasive method of treatment HIFU. The paper describes the mechanism of action and clinical features of the effect of treatment of fibroadenomas of the mammary glands by HIFU. Morphocytology effects confirmation of the method HIFU ablation breast fibroadenoma.
\end{abstract}

Keywords High-intensity Focused Ultrasound (HIFU), Benign Breast Fibroadenoma Breast

\section{The Topicality of the Problem}

Benign breast changes are among the most common diseases in women of different age groups and include a variety of clinical, morphological and etiological characteristics of the process. Benign breast disease in a female population for example in Russian distributed to $30-70 \%$ of the cases [1].In this pathology such changes as epithelial hyperplasia are considered as markers of increased risk of breast cancer [2].It is known that malignant tumors of the mammary glands occur 3-5 times more on the background of benign mammary glands [3].

Fibroadenoma- one of the most common diseases of the mammary glands and the major part of all benign tumors.

One way to reduce morbidity and mortality from breast cancer is the treatment of benign breast diseases. So far, the main treatment is surgery of fibroadenomas. In connection with the development of technology, there were reports on the effective use of high-intensity focused ultrasound-HIFU ablation in the treatment of soft tissue tumors.

HIFU - therapy is possible to avoid the disadvantages of surgical treatment as today it is the only non-invasive method of destruction of education conducted without violating the integrity of the skin. New non-invasive technology to treat tumors based on the use of high-intensity focused ultrasound - HIFU (High Intensive Focused Ultrasound) is a promising direction in medical practice, as evidenced by the growth of publications of authors who use this method [4] [5].

\section{Materials and Methods}

The group of patients was formed from 39 patients with the diagnosis of noncommunicative fibroadenomatosis breast. Age of patients is from 16 to 62 . A diagnosis is based on clinical data, ultrasound, and results of basic biopsy. For realization trephine biopsy used the needle of biopsy. After discussion of all the results was developed algorithm treatment HIFU-therapy. The control group of patients consists of 40 patients with a diagnosis of localized fibroadenomatosis breast. At the age of 16 to 62 years, the patients of control group treated oneself classic by a surgical method.

HIFU -therapy performed using ultrasound system «JC» -Use high-intensity focused ultrasound. Therapeutic ultrasound system «JC» - consists of a transducer disk with a diameter of $12 \mathrm{~cm}$ with aluminum lens (focal length ranged $10-16 \mathrm{sm}$ depending on location) with therapeutic (treatment) ultrasonic frequency of $0.8 \mathrm{MHz}-2.4 \mathrm{MHz}$ and an acoustic frequency in the field of medical damage from $5000 \mathrm{~W} / \mathrm{cm}$ to $25,000 \mathrm{~cm}$. In the central part of the additional transducer is a diagnostic ultrasound scanner with a frequency of $3.5 \mathrm{MHz}$ ultrasound to visualize tissue and areas of therapeutic effects. Therapeutic head with transducer located in a rubber tub. Containing degassed water which is mounted on a village, a gantry attached to the product. This allows the head to rotate relative to the therapeutic focus in three directions in the range of $13 \mathrm{~cm}$ in any direction with precision $+/-0.1 \mathrm{~mm}$. There is a management console that contains three manitora and control panel. On the right monitor is fixed initial state of tissues. On the left dynamically displays the current process of treatment made with color fixation stages of treatment. On the third monitor in the center console displays the position of the table and the medical treatment of the head with the transducer. Separately located the engine room, where there are power system, feeding and degassing. Destruction of high-intensity focused ultrasound fibroadenoma occurs as a result of transformation 
of mechanical energy into thermal energy (the temperature in the focus area reaches $80-100^{\circ} \mathrm{C}$ ) and the cavitation effect (ultrasound vibration causes tissue in which the molecular structure are subjected to compression and decompression of [6-8].

All patients before treatment were carried out following the study of mammary glands:

- Mammografiya

- Ultrasound research

- Morphocytological research

Distribution of patients depending on the location and size of breast fibroadenomas are shown in Table 1.2.

Table 1. Distribution of localization breast fibroadenoma

\begin{tabular}{|c|c|c|}
\hline Localization & Abs. & $\%$ \\
\hline Right & 21 & 53,8 \\
\hline Left & 11 & 28,2 \\
\hline 2 sides & 7 & 18,0 \\
\hline in total & 39 & 100 \\
\hline
\end{tabular}

Patients were divided into three groups depending on the size of fibroadenomas (Table 2).

Table 2. Distribution of the size of the breast Education

\begin{tabular}{|c|c|c|c|}
\hline Groups & $\begin{array}{c}\text { Diameter } \\
(\mathrm{mm})\end{array}$ & Abs. & $\%$ \\
\hline I & $10-20$ & 30 & 65,2 \\
\hline II & $21-30$ & 13 & 28,3 \\
\hline III & $31-50$ & 3 & 6,5 \\
\hline & in total & 46 & 100 \\
\hline
\end{tabular}

\section{The Algorithm of the HIFU-Ablation Breast Fibroadenoma}

Explanatory work with women on the offered treatment method.

Before carrying out medical procedures, all patients meet people and sign the document, "Informed consent to conduct medical actions and possible risks. [9].

HIFU - therapy was performed under intravenous anesthesia. After the start of anesthesia the patient is placed in the desired position for treatment. Ablation performed with focused ultrasound swing in the vertical direction by 5 $\mathrm{mm}$ slices. The power of the radiation intensity was averaged 178,5W. Energy expended was 56560,9 J. Depending on the size and location of breast fibroadenoma. The results were processed biostatistical method of Kaplan-Meier

HIFU - therapy is conducted at the key form of fibroadenoma breakwater.glands by a diameter from $1 \mathrm{~cm}$ to $5 \mathrm{~cm}$ for the women of reproductive age. Procedure is conducted on principle of ambulatory help. Time of procedure depends on the sizes of tumour and location of them. On the average time of procedure occupies 3 minutes. The multipleness of procedure also depends on the sizes of tumour and averages 1-2 times.

\section{The Results}

The control group of patients consists of 40 patients with a diagnosis of localized fibroadenomatosis breast. At the age of 16 to 62 years. The patients of control group treated oneself classic by a surgical method. The patients were in the hospital for 2-3 days after sector resection of the affected area of the breast.

Efficacy of treatment was evaluated accompaniment control ultrasonic imaging study (Figure 1), cytomorphological study (Figure 2), ultrasonography (Figure 3).

Of the 30 patients treated fibroadenomas I group after 6 months after the HIFU - ablation examined 19 patients. In 6 cases, ultrasound scanning the tumor was not visualized in 8 cases, there hyperechogenicity, reducing the diameter of Education 2 times. In 5 cases the size of fibroadenoma is not changed, but changed ehostructure, she became hyperechoic. In group II fibroadenomas treated at 6 months were examined 8 patients, of whom 5 at ultrasonography noted hyperechogenicity, reducing the size of education in 2 times. In 3 cases, there is hyperechogenicity tumor without resizing. In group III, tumor size did not change throughout the ultrasonographic tumor hyperechoic areas (areas of fibrosis). While in a control group for 14 women at that diameter of fibroadenoma more $3,0 \mathrm{~cm}$, a relapse was marked through 8-10 months; for 2 women in 1 year - the fibrotic-cystophorous regeneration of fabric of mammary gland took place on an intact mammary gland. The repeated surgical interference was required, that was stress for women. 


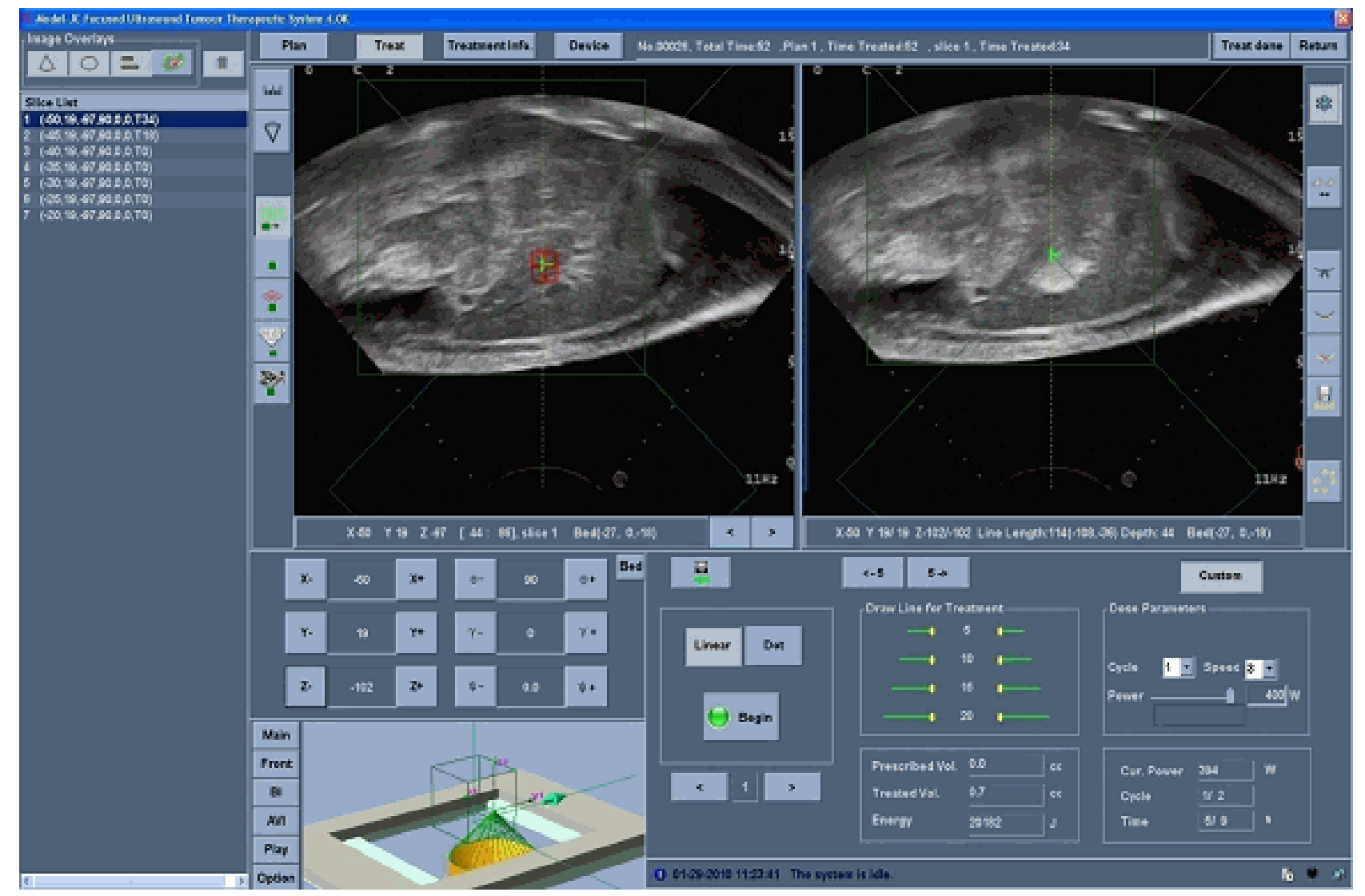

Figure 1. The therapeutic effect in real time gray scale monitor ultrasound guidance. On the right screen hyperechoic plot "a boiling" tumor tissue.
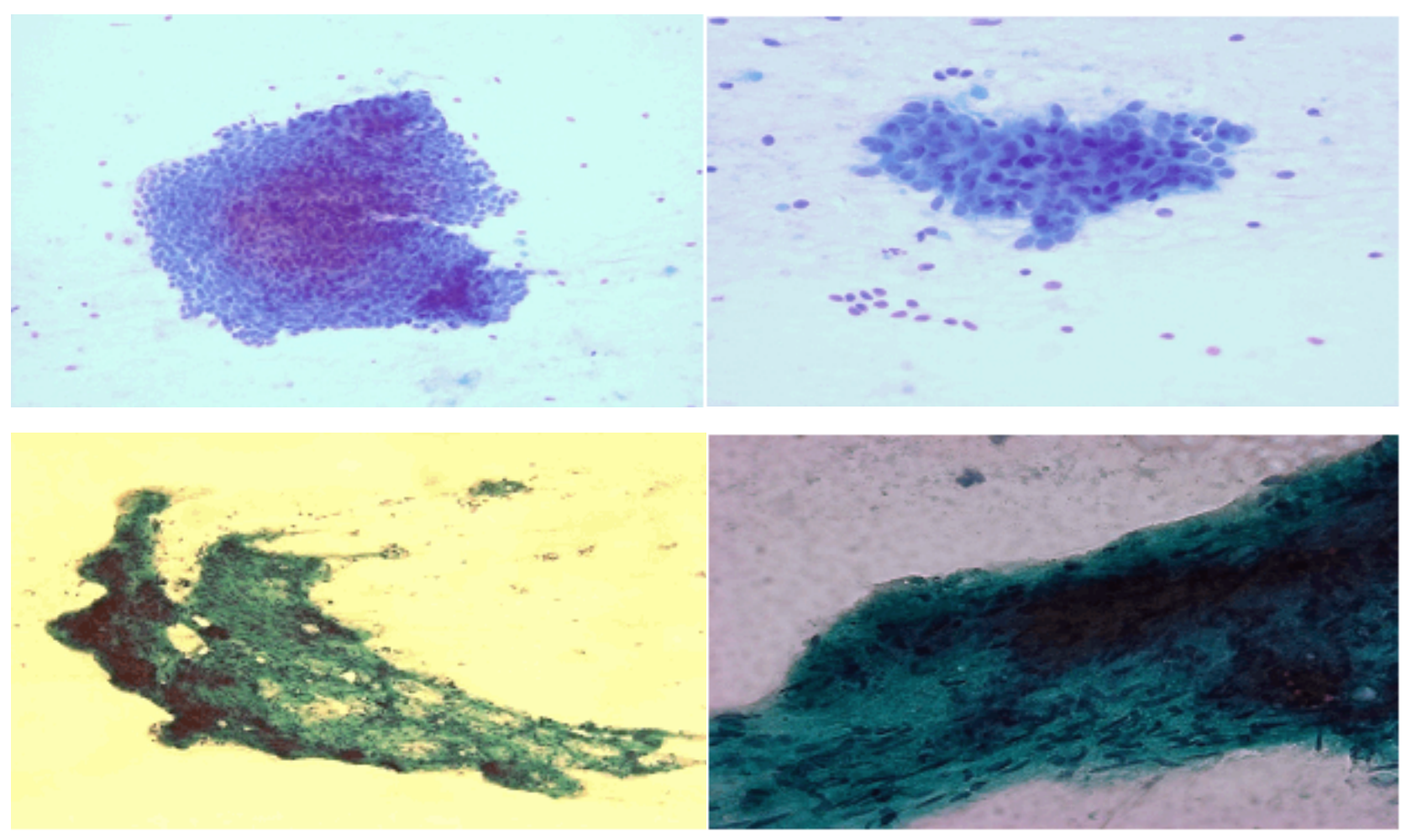

Figure 2. Morphocytological research: A) to HIFU - therapy adenomatous cells; B) after HIFU - therapy, necrosis of cell structures.. 


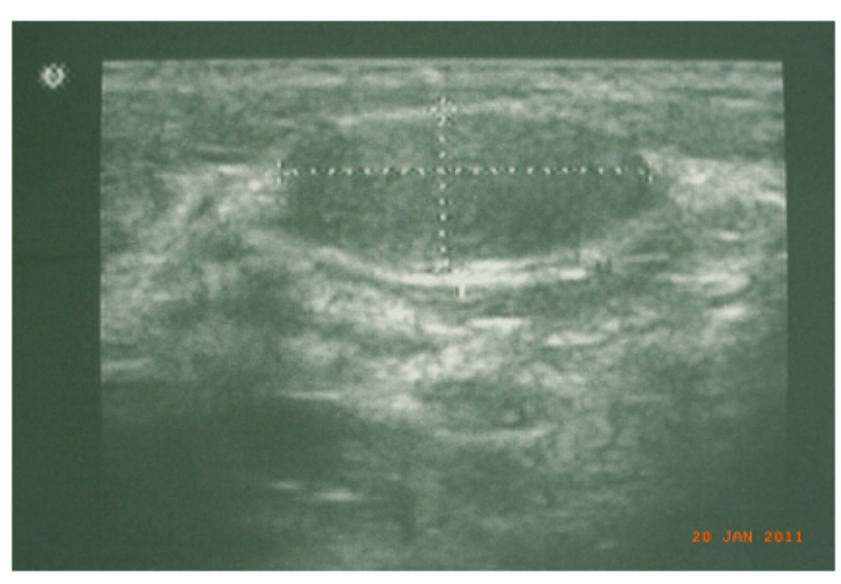

A

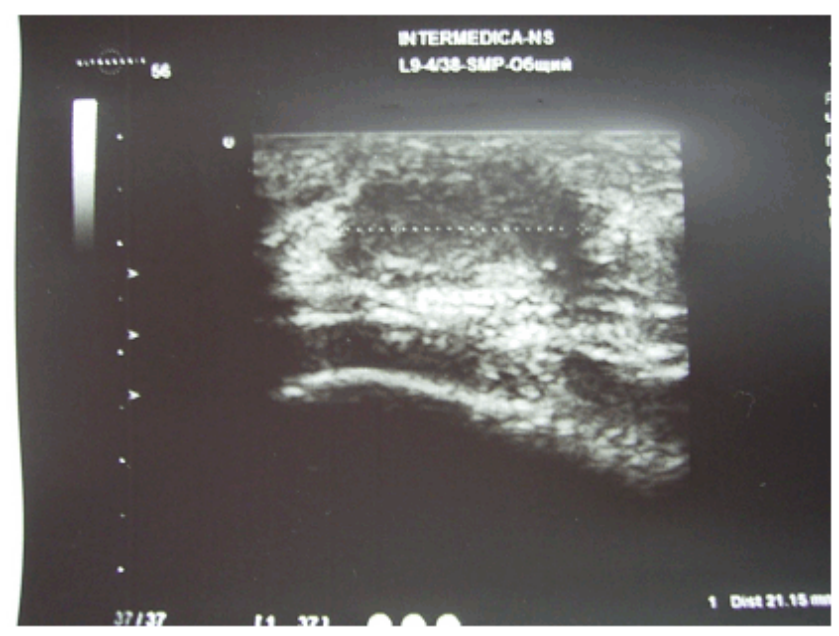

B

Figure 3. Ultrasound research : A) to HIFU - therapy; B) after HIFU therapy fibrozirovannoy hyperechoic tissue structure

\section{Conclusions}

Advantages of the offered methodology in results by comparison to classic surgical method:

- non- invasive;

- economic effect (reduction of stay of days partite in a hospital);

- absence of relapses during 3 years;
- emotional effect (absence of dread of surgical treatment);

- cosmetic effect (there are not scars).

1) HIFU - therapy is an effective method of treating breast fibroadenoma.

2) HIFU - therapy can be used as a separate type of treatment breast fibroadenoma.

\section{REFERENCES}

[1] Semiglazov V.F., Kanaev C.V., Bugrov L.I. Interim results of a randomized study "To evaluate the role of adjuvant radiotherapy in organ-preserving treatment of breast cancer." Questions oncology,1998, p. 436-439.

[2] Shedrina R.N. The hormonal status of the female body during the formation and extinction of reproductive function. Moscow 1986, p. 9-28.

[3] Lindenbraten L.D. The tactics of complex examination in dysplastic and neoplastic lesions of the breast. Surgery 1982, № 2, p. 8-15.

[4] Sickler E. Perodic Mammography Follori-up of Probable benign lesions Result in 3184 Consecutive Cases. Radiol.,1991, 2, 463-468.

[5] Imankulov S.B., Fedotovskich G.V., Asabaev A.S., Erlan M., Shaymardanova G., Zhampeisov N.K."Opportunities for high-intensity focused ultrasound in the treatment of hepatic echinococcosis" Article 6 of the Russian Congress of Association of diagnostic ultrasound in medicine. Moscow 2011;p.130

[6] Kennedy JE, ter Haar GR, Cranston D. High Intensity Focused Ultrasound: surgery of the future. Brit J Radiol,2003,76;590-599.

[7] Hill CR, Rivens I, Vaughan M, et al. Lesion development in focused ultrasound surgery: a general model. Ultrasound Med Biol. 1994;20:259-69

[8] Vaughan M, ter Haar G, Hill CR, et al. Minimally invasive cancer surgery using focused ultrasound: a pre-clinical, normal tissue study. Br J Radiol. 1994;67:267-74

[9] Aykumbekov NR. Human Reproduction and criminal law: problems and judgments. Almata.2007, p.256 